ISSN (Versão Impressa): 1519-9894

ISSN (Versão Online): 2179-2194

\title{
fragmentum
}

\section{- NÚMERO ESPECIAL -}

HISTÓRIA DA CIÊNCIA DA LINGUAGEM, DAS TEORIAS LINGUÍSTICAS, DA CONSTRUÇÃO

DO CONHECIMENTO SOBRE A(S) LÍNGUA(S)

Claudia Castellanos Pfeiffer (LABEURB/NUDECRI - UNICAMP)

Organizadora
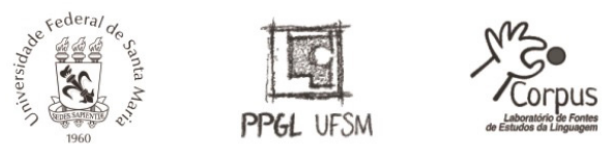

PPGL UFSM 
Fragmentum / Universidade Federal de Santa Maria. Centro de Artes e Letras. Programa de Pós-Graduação em Letras. Laboratório Corpus.

N. 1 (set. 2001) - . Santa Maria, 2001 -.

Disponivel em: https://periodicos.ufsm.br/index.php/fragmentum Semestral

ISSN $1519-9894$ (versão impressa)

e-ISSN 2179-2194 (versão online)

Número especial. "História da ciência da linguagem, das teorias linguísticas, da construção do conhecimento sobre a(s) língua(s)", organizado por Claudia Castellanos Pfeiffer (LABEURB/NUDECRI UNICAMP).

1. Conhecimento sobre a língua 2. História, memória e teorias linguísticas 3. Universidade Federal de Santa Maria (UFSM). 4. Centro de Artes e Letras (CAL).

Ficha catalográfica elaborada por Luciano Rapetti - CRB 10/2031

Biblioteca Central da UFSM

\section{Editora do Programa de Pós-Graduaçáo em Letras}

Programa de Pós-Graduação em Letras - Universidade Federal de Santa Maria

Prédio 16, CE, sala 3222 - Bloco A2

Campus Universitário - Bairro Camobi

CEP 97105-900 - Santa Maria, RS - Brasil

Fones: 5532208359 - 5532208025

Email:ppgletras@ufsm.br

Site: www.ufsm.br/ppgletras

Página no Facebook: PPGL Editores

Fragmentum

www.ufsm.br/fragmentum - fragmentum.corpus@gmail.com

\section{Data da Efetiva Circulaçáo}

Dezembro de 2018

Impresso na Imprensa Universitária da UFSM

Avenida Roraima, 1000 - Prédio 6

Campus Universitário - Bairro Camobi

CEP 97119-900 - Santa Maria - RS

Fone: (55) 3220-8249

\section{Apoio}

Pró-Reitoria de Pós-Graduação e Pesquisa - PRPGP/UFSM - Edital Pró-Revistas
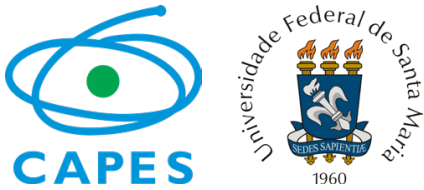

Pró-Reitoria de Extensão - PRE/UFSM 


\begin{abstract}
fragmentum
Publicaçáo do Laboratório Corpus - Laboratório de Fontes de Estudos da Linguagem, do Programa de Pós-Graduação em Letras da UFSM
\end{abstract}

\title{
ANO DA PRIMEIRA PUBLICAÇÃO
}

2001

\section{POLÍTICA EDITORIAL}

Fragmentum é um periódico científico publicado trimestralmente nas versóes impressa (ISSN 1519-9894) e on-line (ISSN 2179-2194) e destinado a pesquisadores e estudantes em nível de pós-graduação. $\mathrm{O}$ periódico divulga textos produzidos por pesquisadores que desenvolvem, como escopo e/ou resultado de pesquisas, as seguintes problemáticas:

a) $\mathrm{Na}$ Linguística, questôes enunciativas e/ou discursivas, tendo por eixo diretor o campo do saber sobre a história da produçáo do conhecimento linguístico, a partir da análise de instrumentos linguísticos bem como de outras textualidades alicerçadas pela História das Ideias Linguística em sua relação com a Análise de Discurso de linha francesa;

b) $\mathrm{Na}$ Literatura, estudos comparados que têm evidenciado a relação do texto literário não apenas com seu contexto de produção como também com outras artes, mídias, saberes e formas, aproximação esta que articula artes e conhecimentos em suas especificidades, demonstrando processos de leitura, compreensão, interpretação e análise envolvidos no acesso a obras de arte e à recepção de um público especializado.

Admitem-se textos em português, francês, inglês ou espanhol. Não são aceitos textos de pesquisadores que não tenham a formação mínima de doutor. Acadêmicos de doutorado podem submeter textos à avaliaçáo, desde que em coautoria com o professor orientador.

Com periodicidade semestral, cada novo dossiê temático será organizado por dois pesquisadores e constituído de um conjunto de artigos somados a uma resenha e à divulgaçáo, em formato de resumo, de duas teses já defendidas, que apresentem relevância para a temática em foco. Afora essa estrutura preestabelecida, Fragmentum se reservará o direito de publicar entrevistas e outras textualidades inéditas, de caráter artístico e ensaístico, quando convier. Originais em francês, português e espanhol deverão apresentar título, resumo e palavras-chave na língua em que foi escrito o texto e em inglês. Para originais em inglês, título, resumo e palavras-chave deverão ser apresentados em inglês e em português. 
Reitor da Universidade Federal de Santa Maria

Paulo Afonso Burmann

Diretor do Centro de Artes e Letras

Cláudio Antônio Esteves

Coordenadora do Programa de Pós-Graduação em Letras

Eliana Sturza

Coordenadora Geral do Laboratório Corpus

Taís da Silva Martins

Larissa Cervo Montagner

\section{Comitê Editorial}

\section{Comissáo Editorial}

Amanda Eloina Scherer, UFSM, Santa Maria, RS, Brasil

Pedro Brum Santos, UFSM, Santa Maria, RS, Brasil

Verli Petri, UFSM, Santa Maria, RS, Brasil

\section{Editora-Chefe}

Amanda Eloina Scherer, UFSM, Santa Maria, RS, Brasil

\section{Editora-Gerente}

Maria Iraci Sousa Costa, UFSM, Santa Maria, RS, Brasil

\section{Editores de Língua Estrangeira}

Francês - Amanda Eloina Scherer, UFSM, Santa Maria, RS, Brasil

Inglês - Enéias Farias Tavares, UFSM, Santa Maria, RS, Brasil

Espanhol - Germán García Bermúdez, Universidad de la República, Montevideo,

Uruguay

\section{Conselho Editorial}

Alcides Cardoso dos Santos, UNESP, Araraquara, SP, Brasil

Ana Zandwais, UFRGS, Porto Alegre, RS, Brasil

Beatriz Maria Eckert-Hoff, UDF, Brasília, DF, Brasil

Bethania Mariani, UFF, Niterói, RJ, Brasil

Caciane Souza de Medeiros, UFSM, Santa Maria, RS, Brasil

† Carme Regina Schons, UPF, Passo Fundo, RS, Brasil

Cristiane Dias, UNICAMP, Campinas, SP, Brasil

Eduardo Guimarães, UNICAMP, Campinas, SP, Brasil

Enéias Farias Tavares, UFSM, Santa Maria, RS, Brasil

Eni Puccinelli Orlandi, UNICAMP, Campinas, SP, Brasil

Flavio Felicio Botton, UFABC, Santo André, SP, Brasil

Flávio Loureiro Chaves, UFRGS, Porto Alegre, RS, Brasil 
Gema Sanz Espinar, Universidad de Madrid, Madrid, Espanha

Gerson Luiz Roani, UFV, Viçosa, SP, Brasil

Gesualda Rasia, UFPR, Curitiba, PR, Brasil

Gisela Biancalana, UFSM, Santa Maria, RS, Brasil

Gladys B. Morales, Universidad Nacional de Río Quarto, Argentina

Héliane Kohler, Université de Franche-Comté, França

Irène Fenoglio, Centre National de la Recherche Scientifique, Paris, França

Isabel Cristina Ferreira Teixeira, UNIPAMPA, Bagé, RS, Brasil

José Horta Nunes, UNICAMP, Campinas, SP, Brasil

José Luís Jobim de Salles Fonseca, UFRJ, Rio de Janeiro, RJ, Brasil

Juan Manuel López Muñoz, Universidad de Cadiz, Cadiz, Espanha

Juliana Steil, UFPEL, Pelotas, RS, Brasil

Larissa Montagner Cervo, UFSM, Santa Maria, RS, Brasil

Lucília Maria Sousa Romão, USP-Ribeirão, Ribeirão Preto, SP, Brasil

Maria Cleci Venturini, UNICENTRO, Guarapuava, PR, Brasil

Maria da Glória Bordini, UFRGS, Porto Alegre, RS, Brasil

Maria José R. Faria Coracini, UNICAMP, Campinas, SP, Brasil

Marilene Weinhardt, UFPR, Curitiba, PR, Brasil

Mary Neiva Surdi da Luz, UFFS, Chapecó, SC, Brasil

Orna Messer Levin, UNICAMP, Campinas, SP, Brasil

Paulo Ricardo Kralik Angelini, PUCRS, Porto Alegre, RS, Brasil

Regina Zilberman, UFRGS, Porto Alegre, RS, Brasil

Silmara Dela Silva, UFF, Niterói, RJ, Brasil

Taís da Silva Martins, UFSM, Santa Maria, RS, Brasil

Vanise Gomes de Medeiros, UFF, Niterói, RJ, Brasil

Véronique Daleth, USP, São Paulo, SP, Brasil

\section{Produção Editorial}

\section{Capa e Projeto Gráfico Originais}

Mirian Rose Brum-de-Paula, UFPEL, Pelotas, RS, Brasil

Simone de Mello de Oliveira, UFSM, Santa Maria, RS, Brasil

\section{Produçáo Gráfica}

João Marco de Abreu Christo, UFSM, Santa Maria, RS, Brasil Júlia Carmelli Campos, UFSM, Santa Maria, RS, Brasil 


\section{Editoraçáo Eletrônica}

Marcos Soares, UFSM, Santa Maria, RS, Brasil

\section{Revisáo}

Andressa Brenner, UFSM, Santa Maria, RS, Brasil

Bruna Cielo Cabrera, UFSM, Santa Maria, RS, Brasil

Kelly Guasso, UFSM, Santa Maria, RS, Brasil

Viviane Teresinha Biacchi Brust, UFSM, Santa Maria, RS, Brasil

\section{Indexadores}

Google Acadêmico

Latindex - Sistema Regional de Información en Línea para Revistas Científicas de América Latina, el Caribe, Espańa y Portugal

Portal de Periódicos da CAPES

Rede Cariniana (IBICT)

Portal de Periódicos da UFSM

WorldCat ${ }^{\circ}$ (OCLC)

EZB - Elektronische Zeitschriftenbibliothek 